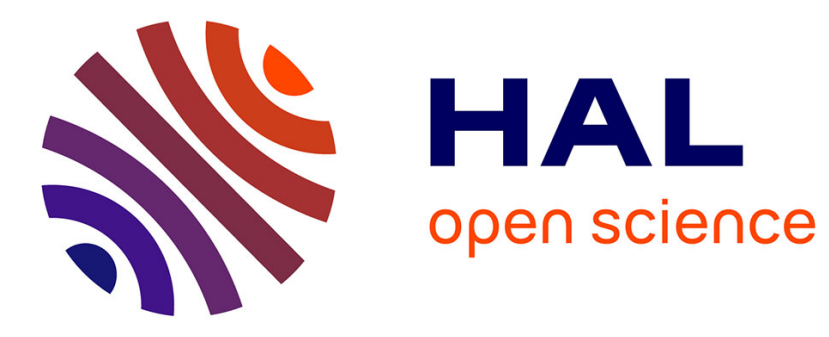

\title{
Chemical Shift Imaging (CSI) by precise object displacement
}

Sebastien Leclerc, Gregory Trausch, Benoit Cordier, Denis Grandclaude, Alain Retournard, Jacques Fraissard, Daniel Canet

\section{- To cite this version:}

Sebastien Leclerc, Gregory Trausch, Benoit Cordier, Denis Grandclaude, Alain Retournard, et al.. Chemical Shift Imaging (CSI) by precise object displacement. Magnetic Resonance in Chemistry, 2006, 44 (3), pp.311-317. 10.1002/mrc.1757 . hal-00124995

\section{HAL Id: hal-00124995 \\ https://hal.science/hal-00124995}

Submitted on 17 Jan 2007

HAL is a multi-disciplinary open access archive for the deposit and dissemination of scientific research documents, whether they are published or not. The documents may come from teaching and research institutions in France or abroad, or from public or private research centers.
L'archive ouverte pluridisciplinaire HAL, est destinée au dépôt et à la diffusion de documents scientifiques de niveau recherche, publiés ou non, émanant des établissements d'enseignement et de recherche français ou étrangers, des laboratoires publics ou privés. 


\title{
Chemical Shift Imaging (CSI) by precise object displacement**
}

\author{
Short title: NMR lift
}

Sebastien Leclerc $^{(\mathrm{a})_{\text {,(b) }}}$, Gregory Trausch ${ }^{(\mathrm{a})}$, Benoit Cordier $^{(\mathrm{a})}$, Denis Grandclaude ${ }^{(\mathrm{a})}$, Alain Retournard $^{(\mathrm{a})}$, Jacques Fraissard ${ }^{(\mathrm{c})}$, Daniel Canet*(a)

${ }^{(a)}$ Méthodologie RMN (UMR 7565 CNRS-UHP), Université Henri Poincaré, Nancy I, B.P. 239

54506-Vandoeuvre-les-Nancy (cedex), France

(b) LEMTA (UMR 7563 CNRS-INPL-UHP), B.P. 160

54504-Vandoeuvre-les-Nancy (cedex), France

${ }^{(c)}$ Laboratoire Physique et Mecanique des Milieux Hétérogènes (LPMMH), ESPCI and

Université P. et M. Curie, 10 rue Vauquelin

75231-Paris (cedex 05), France

* Daniel.Canet@rmn.uhp-nancy.fr, phone : 333836843 49, fax : 33383684347

**: presented in part at the Ampere XI NMR School, Zakopane (Poland); June 1-6, 2003

Abstract : A mechanical device (NMR lift) has been built for displacing vertically an object (typically a NMR sample tube) inside the NMR probe with an accuracy of $1 \mu \mathrm{m}$. A series of single pulse experiments are performed for incremented vertical positions of the sample. With a sufficiently spatially selective rf field, one obtains chemical shift information along the displacement direction (one dimensional Chemical Shift Imaging - CSI). Knowing the vertical radio-frequency (rf) field profile (the amplitude of the $\mathrm{rf}$ field along the vertical direction) makes it possible to reconstruct the spectrum associated with all the slices corresponding to consecutive sample positions and to improve the spatial resolution, which is simply related to the accuracy of the displacement device. Besides tests performed on phantoms, the method has been applied to solvent penetration in polymers and to benzene diffusion in a heterogeneous zeolite medium.

Keywords : NMR, CSI, ${ }^{1} \mathrm{H}$, mechanical device, imbibed polymer, zeolite, diffusion 


\section{Introduction}

Several NMR experiments make use of mechanical devices whenever manipulations of nuclear magnetization cannot be achieved by appropriate radio-frequency field or magnetic field gradient pulses. The most popular procedure is the so-called magic angle spinning (MAS) which reduces or averages out some anisotropic interactions which are involved in solid state NMR. In this respect, the FIREMAT experiment ${ }^{1}$ which aims at determining chemical shift tensor principal values requires to synchronize very precisely the pulse sequence with the rotor position. This experiment employs an optical equipment for such a control; along the same lines, we wish to present here an instrumental arrangement by which the vertical position of the sample is settled in a very precise way, the aim being to obtain the NMR spectrum in as many thin slices as fixed by the step-by-step displacement of the sample. This is nothing less than one-dimensional chemical shift imaging $(\mathrm{CSI})^{2}$ with a spatial resolution not dictated by the magnetic field gradient amplitude or by the corresponding pulse sequence but rather by the accuracy of the sample displacement combined with the spatial selectivity of the radio-frequency field ( $\mathrm{rf}$ field or $\mathrm{B}_{1}$ field) employed in the relevant NMR experiment. Of course, sample (or object) displacement in NMR imaging or microscopy is not new. This is a common practice in Stray Field Magnetic Resonance Imaging (STRAFI) ${ }^{3,4}$. However, in our case, we shall not rely on gradients of the static magnetic field ( $\mathrm{B}_{0}$ field) but rather take advantage of the inhomogeneity of the radio-frequency field generated by the rf coil of the probe in a vertical cryomagnet ${ }^{5}$. This coil is saddle shaped and its geometry has been purposely altered with respect to the classical design ${ }^{6}$ in order to increase its natural spatial selectivity.

The interest of this strategy with respect to conventional CSI would be to increase both spectral and spatial resolutions. Concerning spectral resolution, this results simply from the fact that successive standard high resolution spectra are acquired with non imaging equipment 
and without switching gradients, thus maintaining the usual performances of high resolution NMR. On the other hand, spatial resolution is expected to be improved by means of proper experimental conditions, as explained above.

The aim of the method is thus to determine the evolution of the NMR spectrum in a heterogeneous sample. It will be checked with model samples and then applied to problems of chemical interest, namely solvent penetration in polymers and kinetics of gaseous benzene adsorption into a zeolite.

\section{General Principle, instrumentation and raw images}

The idea is to select successively slices at different positions of the sample along the vertical $\mathrm{Z}$ axis which corresponds to the direction of the static magnetic field when dealing with a vertical cryomagnet. In this way one obtains a spectrum corresponding to the composition of the sample at the relevant position and this could be of some interest in the case of heterogeneous samples. In order to perform such experiments, a device (to be detailed below) was designed so as to displace the sample by increments of the order of the micrometer at the lowest.

Figure 1

As schematized in figure 1, spatial selectivity is achieved primarily by the rf field excitation profile which is generally much broader than the displacement increment. We can expect a spatial resolution of the order of this displacement increment but this goal will be better reached if the data are processed in a proper way so as to remove the effects of the overlap of the successive excitation profiles corresponding to the incremented sample displacement. A dedicated algorithm is described later. For the moment, we shall concentrate on the displacement device. Its main part is a stepper motor installed at the top of the vertical cryomagnet (see figure 2). 
Figure 2

It is driven by pulses generated by the interface described below and a series of gears (figure 3) which transform rotation into a translation motion applied to an endless screw.

Figure 3

The problem one is faced to is that the vertical displacement is not accurate enough due to errors arising from the electromagnetic environment. As a consequence, the vertical displacement has to be controlled by an independent optical system which acts as a feed-back for correcting the actual motion of the endless screw. Basically, as the optical encoder is of the incremental type, it produces a pulse each time it detects a $1 \mu \mathrm{m}$ displacement. Thus a displacement by $100 \mu \mathrm{m}$ would imply 100 pulses. The electronic device counts the number of pulses and activates the stepper motor until the setting value is reached. In practice, this dedicated electronic device is connected to the main computer via Ethernet and a microcontroller ${ }^{9}$. The logical module is essentially made of a field programmable gate array (fpga) ${ }^{10}$ which activates all logical lines necessary to the control of the sample position (pulses for the stepper motor, optical encoder pulse counter, limit switch signals, zero setting of the optical encoder). It communicates with the main computer to trigger the displacement apparatus and to provide the status of this device.

All experiments presented here concern proton NMR (at $200 \mathrm{MHz}$ ) and were performed at ambient temperature with a home-made console (hardware and software) operated in conjunction with a wide-bore Oxford $4.7 \mathrm{~T}$ magnet (see figure 2). The probe is equipped with a saddle coil of $8 \mathrm{~mm}$ diameter and $4 \mathrm{~mm}$ height.

As the spatial resolution is governed by the spatial selectivity of the rf field, it is important to know the $\mathrm{B}_{1}$ profile along the vertical direction. It is determined by running the experiment described above (a single pulse experiment for each sample position) with a Shigemi tube ${ }^{11}$ containing a drop of water so that the liquid thickness is about $1 \mathrm{~mm}$. This experiment yields 
what we call the "signal profile" which is not exactly the " $\mathrm{B}_{1}$ profile" as explained now: provided that the pulse duration $\tau$ is smaller or equal to that corresponding to the $\pi / 2$ pulse for the region of maximum rf field amplitude, we can write the signal amplitude at a given location $Z$ as $k x \sin x$ where $k$ is a factor independent of $Z$ and $x$ the flip angle $\left(x=\not B_{1}(Z) \tau\right)$. In $k x \sin x$, the factor $x$ arises from the signal receptivity which, by virtue of the reciprocity principle, is proportional to $B_{1}$. Each data point yields a value for $x$ and the plot of $x$ versus $Z$ provides the required profile. In practice we can use the "natural" profile of the rf coil or a profile obtained by slice selection procedures as described in a previous work ${ }^{8}$.

As a first model system, we considered a NMR tube containing two non-miscible liquids (water and toluene)

Figure 4

As these two liquids have very different surface tensions, a meniscus forms at the interface between the two liquids. On the map of figure 4 , which is a simple contour plot of the spectra corresponding to the successive sample displacements, one can see that toluene and water are present simultaneously in a zone which is $4 \mathrm{~mm}$ high, whereas the meniscus is about $1.5 \mathrm{~mm}$ high. This is clearly due to the width of the excitation profile, and could be improved by the reconstruction algorithm (which was not yet implemented at the time of this first experiment). Several interesting phenomena can nevertheless be observed: firstly, it can be seen that the resonance frequencies of the toluene peaks (and to a lesser extent, those of water) increase (shift to the left) when one moves towards the lower part of the sample. This phenomenon is due to an internal static magnetic field arising from the difference in magnetic susceptibility between the glass of the bottom of the tube and the toluene. This internal field is in the opposite direction to the field $\mathrm{B}_{0}$ of the magnetization and increases as one approaches the bottom of the tube. One can also observe on this map the variation of the resonance frequency 
at the interface between the two liquids: this results in a line broadening for the water peak and extra peaks for toluene (this is particularly visible for the methyl resonance).

Figure 5

The next system which was investigated is a piece of high density polyethylene which has been in contact with a mixture of solvents on one of its side. The objective of the experiment is to study the penetration of each component of the mixture into the polymer. From figure 5 , it is seen that, for this contact time ( center of the sample. Some other valuable information can be gained from the examination of line intensities in spectra obtained at different heights: it is clear that isooctane does not enter this polymeric material while ethanol penetrates more slowly (it is present only at the center of the sample) and presumably evaporates more quickly than toluene from the side which is not in contact with the solvent mixture.

\section{Reconstruction algorithm and selected applications}

Although the device allows sample displacements as low as one micrometer, steps of $50 \mu \mathrm{m}$ or $100 \mu \mathrm{m}$ are generally used. These latter values are anyway considerably smaller than the width of the (experimentally determined) signal profile, which is generally between 0.5 and 4 $\mathrm{mm}$. The spectrum obtained corresponds therefore to the partial overlap of several tens of elementary slices (by elementary slice we mean a slice of thickness equal to the step of displacement). The aim of the algorithm described below is to reconstruct the spectrum corresponding to each of these elementary slices from the spectra measured for all the sample positions (the number of sample positions is, by definition, equal to the number of elementary slices). This is related to a procedure which was proposed some time ago to correct distortion effects in images produced by a surface coil of a particular geometry ${ }^{12}$.

Figure 6 
Let $\mathrm{n}$ be the number of displacements of the sample and $\mathrm{m}$ the number of elementary slices in an excitation profile (for example, if the displacement is $100 \mu \mathrm{m}$ and the width of the excitation profile is $1 \mathrm{~mm}$, then $\mathrm{m}=10$ ). $E_{1}$ to $E_{n}$ are the n spectra obtained from successive sample displacements. $S_{1}$ to $S_{n}$ are the spectra we wish to obtain at the end of the reconstruction process, each of them corresponding to a given elementary slice. The excitation profile is first decomposed into $\mathrm{m}$ elementary slices, $P_{k}$ ( $k=1$ to $\left.\mathrm{m}\right)$ being the height of the excitation profile in the middle of the $k^{\text {th }}$ slice (figure 4). As we shall be dealing with an iterative process, we shall denote by $S_{i}(j)$ the reconstructed spectrum for the $i^{\text {th }}$ elementary slice at the $j^{\text {th }}$ iteration. We choose to define the initial spectra $S_{i}(0)$ as (which amounts to identify, as a first approximation, $S_{i}$ to $E_{i}$ )

$$
S_{i}(0)=\frac{E_{i}}{\sum_{k=1}^{m} P_{k}}
$$

while the measured spectrum $E_{i}$ can be expressed, in a general way, as

$$
E_{i}=\sum_{k=1}^{m} S_{k+i-1} \times P_{k}
$$

For example, for $m=3$, we have:

$$
\begin{aligned}
& E_{1}=S_{1} \times P_{1}+S_{2} \times P_{2}+S_{3} \times P_{3} \\
& E_{2}=S_{2} \times P_{1}+S_{3} \times P_{2}+S_{4} \times P_{3}
\end{aligned}
$$

From equation (2) and from the experimental spectrum $E_{i}$, we can derive, at the first iteration a new spectrum $S_{i}$ (which should represent an improvement with respect to the previous one)

$$
S_{i}(1)=\frac{1}{P_{1}}\left[E_{i}-\sum_{k=2}^{m} S_{k+i-1}(0) \times P_{k}\right]
$$

For the example of equations (3), we obtain 


$$
\begin{aligned}
& S_{1}(1)=\frac{1}{P_{1}}\left[E_{1}-S_{2}(0) \times P_{2}-S_{3}(0) \times P_{3}\right] \\
& S_{2}(1)=\frac{1}{P_{1}}\left[E_{2}-S_{3}(0) \times P_{2}-S_{4}(0) \times P_{3}\right]
\end{aligned}
$$

These equations can be generalized for the $j^{\text {th }}$ iteration:

$$
S_{i}(j)=\frac{1}{P_{1}}\left[E_{i}-\sum_{k=2}^{m} S_{k+i-1}(j-1) \times P_{k}\right]
$$

The iterative process will be stopped when two series of spectra corresponding to two successive iterations $S(j)$ and $S(j+1)$ are very close:

$$
\sum_{i=1}^{n}\left|S_{i}(j+1)-S_{i}(j)\right|<\varepsilon
$$

where $\varepsilon$ is a threshold value set according to the precision required.

In practice, all calculations are performed for all the $p$ data points which define a spectrum. The amplitude at the $t^{t h}$ point in the frequency domain will thus be denoted as $S_{i}^{l}$ and the test (7) is actually written as

$$
\sum_{l=1}^{p} \sum_{i=1}^{n}\left|S_{i}^{l}(j+1)-S_{i}^{l}(j)\right|<\varepsilon
$$

The above algorithm, although basic, proved to be useful as shown in the forthcoming example.

In order to check this algorithm with a perfectly flat interface and because we were unable to find a two-phase system without a meniscus, we decided to impregnate a piece of natural rubber with toluene, to place it at the bottom of a $5 \mathrm{~mm}$ diameter tube and to put this tube inside another flat-bottomed tube containing water. From figure 7 , it can first be noticed that 
the resonance frequency shifts (in other words, internal gradients) are less pronounced here (flat interface) than with an interface constituted of a meniscus. No procedure for slice selection of the section was applied, and we simply repeated, for different displacements, an experiment consisting of a $\pi / 2$ pulse followed by signal acquisition. Reconstruction was carried out on the basis of the experimentally determined signal profile and required 28 iterations. The results are presented in figure 7.

\section{Figure 7}

The effect of reconstruction is particularly visible: even if there is still a zone where the two solvents overlap (which does not really exist), the intensity of the peaks in this zone has decreased considerably (by a factor of 2 to 3 ) without the intensity of the peaks being affected at the points where there should be no overlap (for example, inside the rubber). These results confirm then that, although imperfect (with respect to the thickness of the $\mathrm{B}_{1}$ profile and the displacement step), the reconstruction procedure nevertheless leads to a significant improvement in the quality of the plots obtained. The residual unwanted traces arise probably from the inaccuracy of the signal profile used in the reconstruction algorithm.

This reconstruction procedure has been applied to a problem of more chemical concern and deals with gaseous benzene penetration into a zeolite. The simple NMR tube used in the previous experiments is replaced by a more elaborated device, still of axial symmetry, but including several compartments such that the penetration of benzene in powdered zeolite can be triggered at a moment chosen by the experimenter. CSI experiments have been run every 30 minutes. Each experiment involves a total displacement of $1 \mathrm{~mm}$ (corresponding to the height of the compartment containing the zeolite powder) with $50 \mu \mathrm{m}$ increments. Some typical diffusion curves deduced from these experimental data are displayed in figure 8 .

Figure 8 
As expected, one can noted a more rapid diffusion in the upper part of the zeolite compartment along with, at long times, a gas accumulation at the bottom of the compartment. Diffusion rates deduced from these experiments have been checked with an appropriate theory and will be the subject of a work published elsewhere.

\section{Conclusion}

This work has shown that a relatively simple mechanical device combined with a spatially selective radio-frequency field (obtained by a proper geometry of the NMR rf coil) can lead to significant CSI results. For most practical applications, major trends will be accessed by raw data, i.e. evolution of the NMR spectrum as a function of the location inside the object. Such acceptable results can be obtained due to an excitation profile already selective by itself thanks to appropriate saddle coil design. If precise details are needed, it is possible to run a reconstruction algorithm taking into account the overlap of spectra for successive displacements of the sample. The results obtained in the present paper are rather encouraging if compared to conventional CSI, especially as far as spectral resolution is concerned. A limitation of the method comes however from spectral broadening due to internal gradients which appear at interfaces, although these features can yield interesting information related precisely sample inhomogeneity. Anyway, we can anticipate improved chemical shift imaging with more elaborate reconstruction algorithms.

\section{Acknowledgments}

We are grateful to Professor Michael Petryk, from Ternopyl (Ukraine) for theoretical diffusion rate calculations which are in agreement with the curves of figure 8 . 


\section{References}

1. Alderman DW, McGeorge G, Hu JZ, Pugmire RJ, Grant DM. Molec. Phys. 1998; 95: 1131126.

2. Blümich B .: NMR imaging of materials, Clarendon Press: Oxford 2000.

3. Mc Donald PJ. Progr. Nucl. Magn. Reson. Spectrosc. 1997; 30: 69-100.

4. Mc Donald PJ, Newling B, Rep. Prog. Phys. 1998; 61: 1441-1493.

5. Hoult DI, Chen CN, Sank VG, Magn. Reson. Med. 1984; 1: 339-353

6. Hoult DI, Richards RE. J. Magn. Reson. $1976 ; 24$ : 71-85.

7. Maffei P, Mutzenhardt P, Retournard A, Diter B, Raulet R, Brondeau J, Canet D. J. Magn. Reson. 1994 ; A107 : 40-49.

8. Canet D, Leclerc S, Rocher M, Guenneau M, Grandclaude D. Appl. Magn. Reson. 2002 ; $22: 307-318$

9. Chipset SC12-RTOS, Beck@

10. Altera®Max®EPM7128SLC84, ALTERA Corporation.

11. Symmetrical microtube matched with $\mathrm{D}_{2} \mathrm{O}$, SHIGEMI Co. Ltd, Tokyo

12. Baril N, Thiaudière E, Quesson B, Delalande C, Canioni P, Franconi JM J. Magn. Reson. $2000 ; 146,223-227$ 


\section{Figure captions}

Figure 1. Principle of the experiment. The excitation profile corresponds to the rf field $\left(\mathrm{B}_{1}\right)$ produced by the saddle coil surrounding the sample.

Figure 2. Photograph of the upper part of a $4.7 \mathrm{~T}$ wide-bore cryomagnet with the principal elements constituting the NMR lift. The additional stepper motor is for sample rotation used in $\mathrm{rf}_{\text {gradient imaging }}{ }^{7}$.

Figure 3. Simplified scheme of the displacement system. The limit switches prevent to go above and beyond the upper and lower sample positions, respectively. The optical encoder determines accurately the sample position and possibly applies an appropriate correction.

Figure 4. CSI map of a $5 \mathrm{~mm}$ o.d. NMR tube filled with two non-miscible liquids (water and toluene). The central trace corresponds to the water resonance while the two outer traces correspond to the methyl (right) and aromatic (left) toluene resonances. This map is obtained by a series of 100 experiments with a vertical displacement of $100 \mu \mathrm{m}$ between two consecutive experiments. The height of the meniscus is about $1.5 \mathrm{~mm}$, while the overlap of water and toluene resonances is about $4 \mathrm{~mm}$ due to the width of the excitation profile and to the lack of application of the reconstruction algorithm. Each spectrum corresponds to a simple $\pi / 2$ pulse followed by signal acquisition.

Figure 5. CSI map obtained in the same conditions as in figure 4 for a piece of HDPE (high density polyethylene) which has been in contact with a mixture of solvents (isooctane: $45 \%$; ethanol: $10 \%$; toluene: $45 \%$ ) for 6 weeks. The upper part of the figure corresponds to the side exposed to solvents. The three spectra are illustrative of the solvent composition at typical locations in the polymer thickness. Spectra are broadened due to the sample heterogeneity.

Figure 6. Left : Profile of the $B_{1}$ field approximated by three elementary slices $(m=3)$. Right : definition of $E_{i}$ (signal profile) and $S_{i}$ (elementary slices) involved in the reconstruction algorithm $(\mathrm{m}=3)$.

Figure 7. Demonstration of the interest of the reconstruction procedure for a sample of the same nature as in figure 4 but involving a flat interface. Raw data, displayed on the left, show a non-negligible (and unwanted) overlap of toluene with water. This overlap almost disappears after the reconstruction procedure (right).

Figure 8. Diffusion curves of gaseous benzene into powdered ZSM5 zeolite at different locations. $0 \mathrm{~mm}$ corresponds to the part in contact with benzene. 


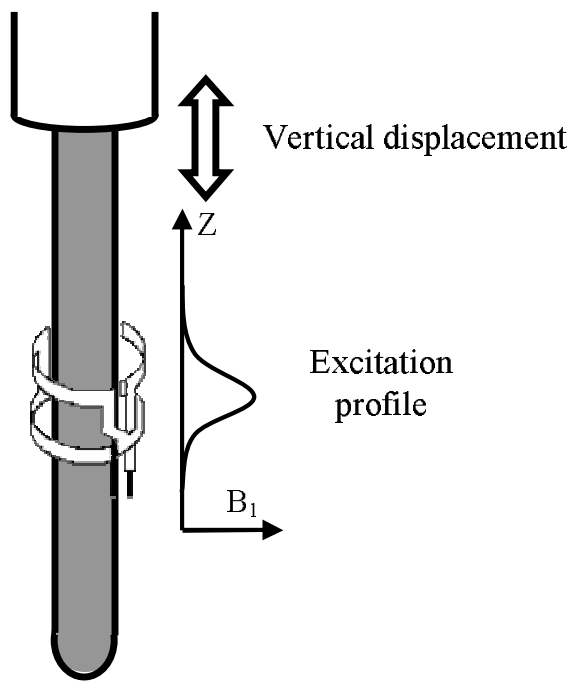

Figure 1 


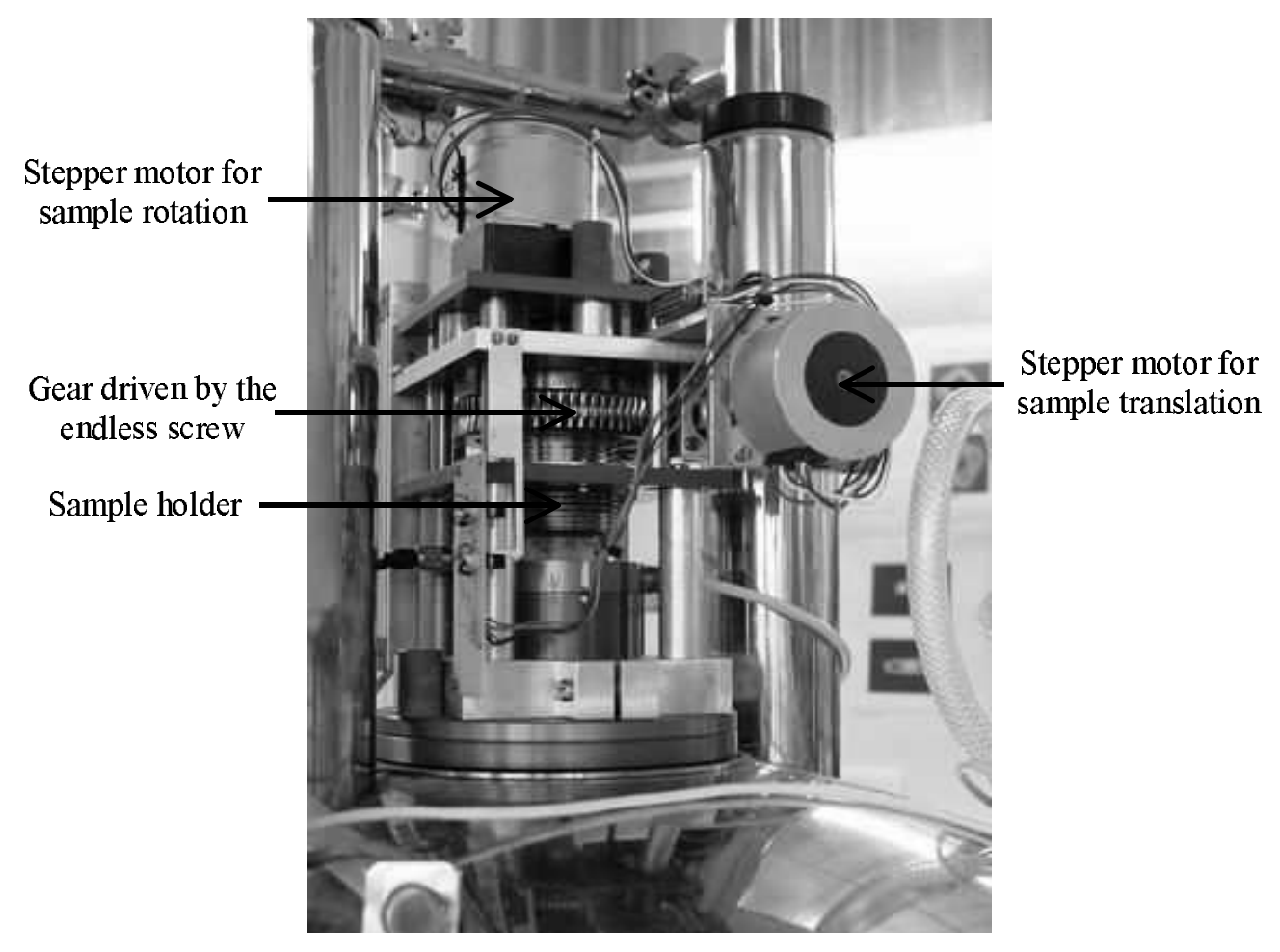

Figure 2 
Endless screw with hollow gear

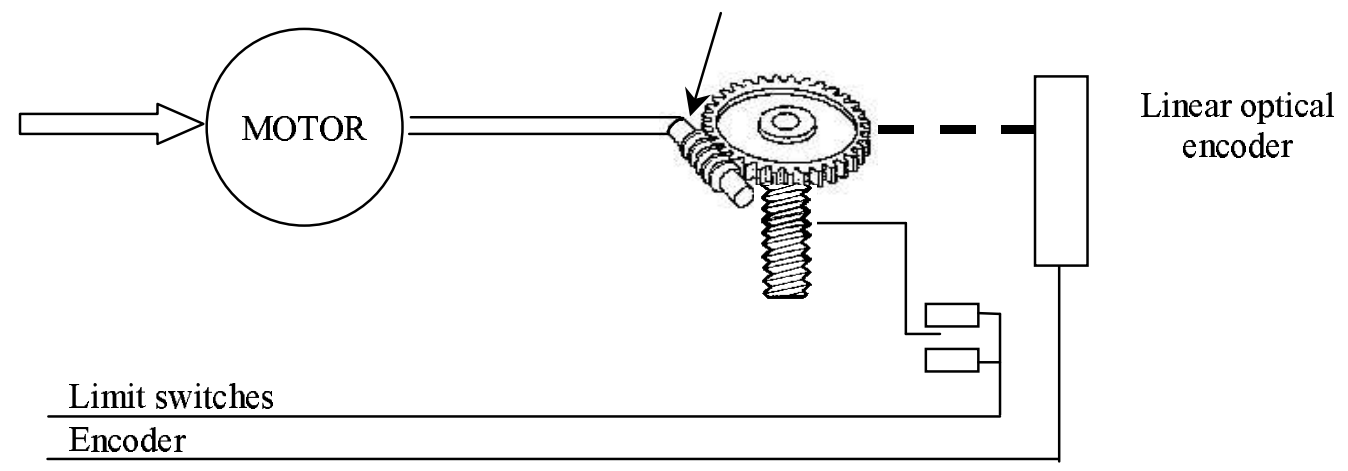

Figure 3 


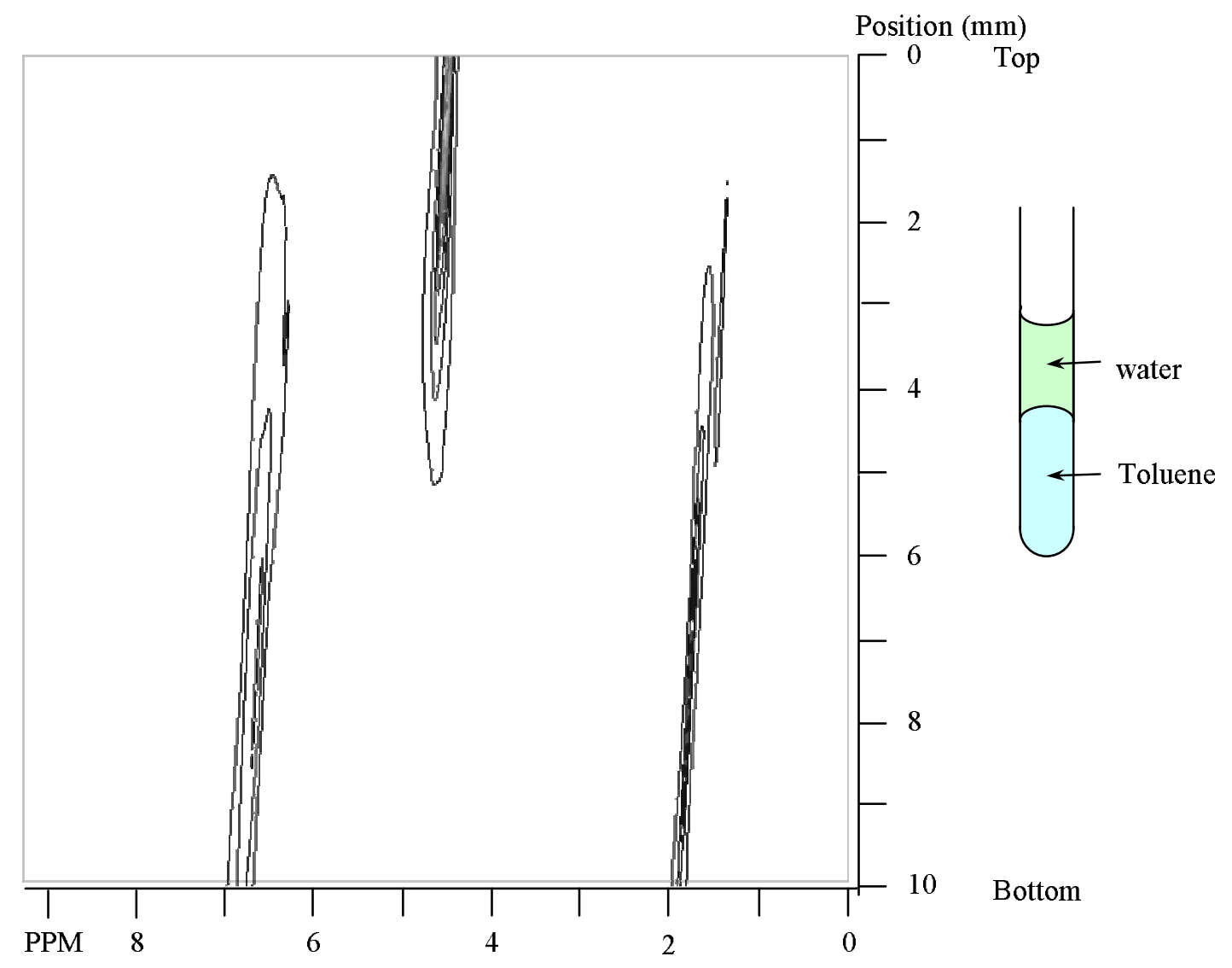

Figure 4 


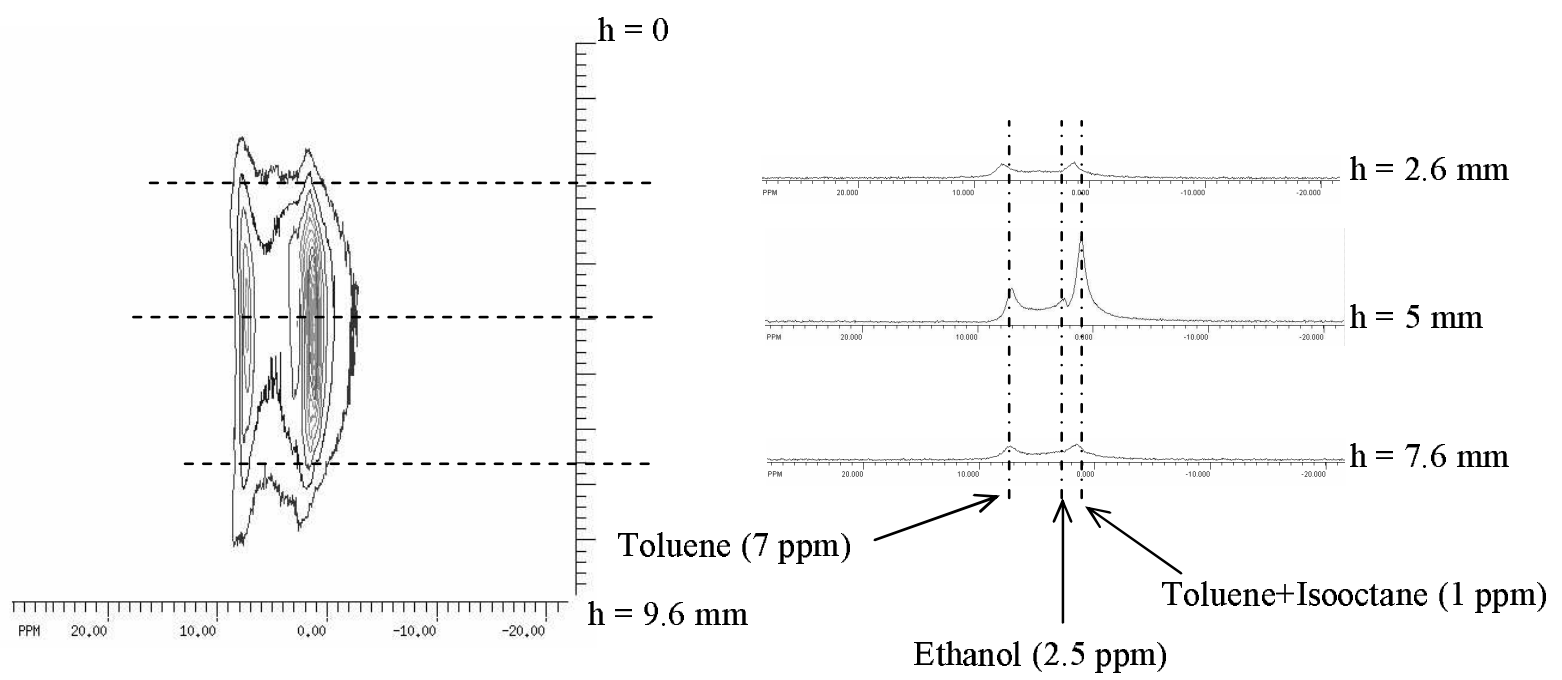

Figure 5 

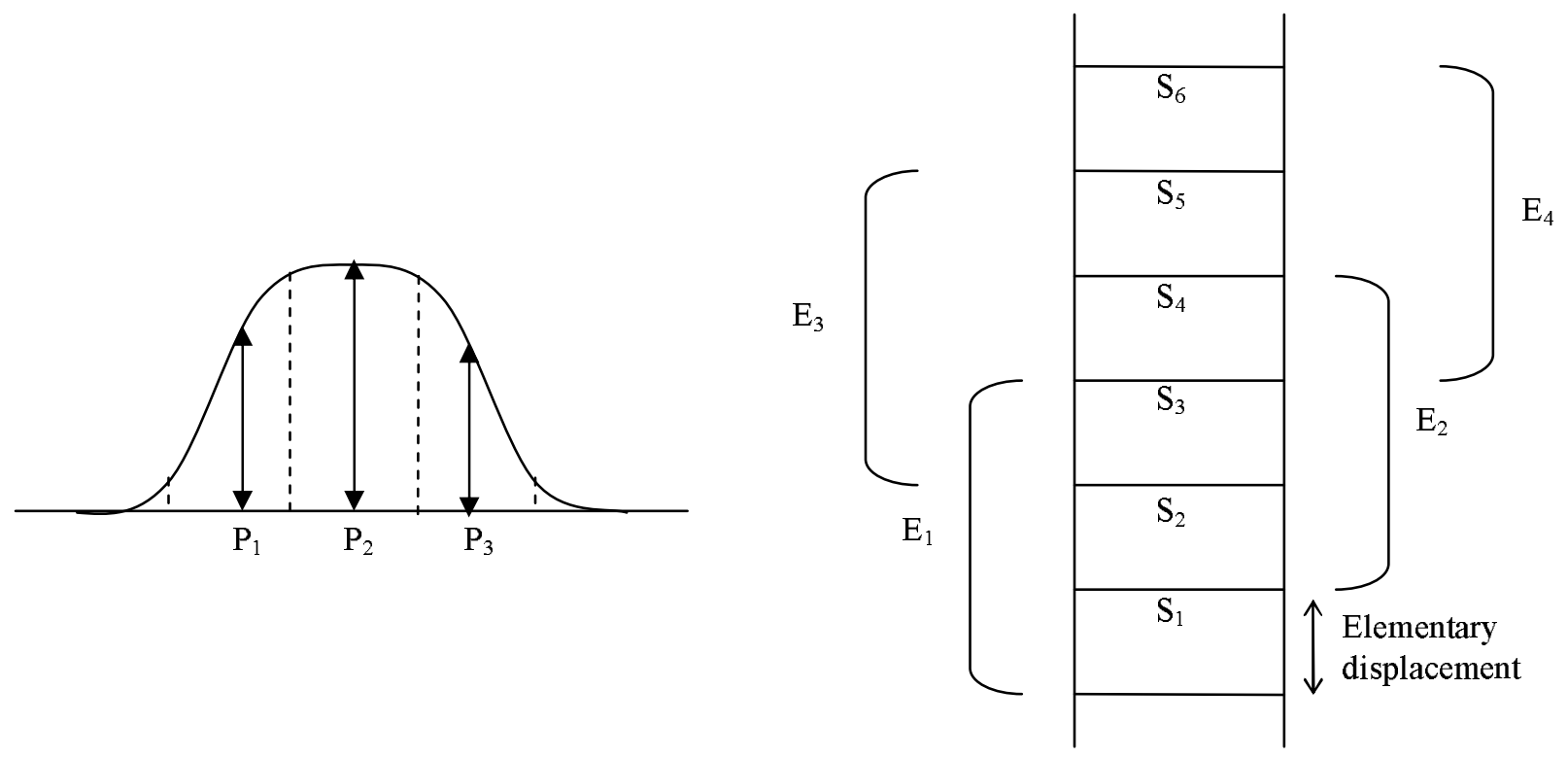

Figure 6 

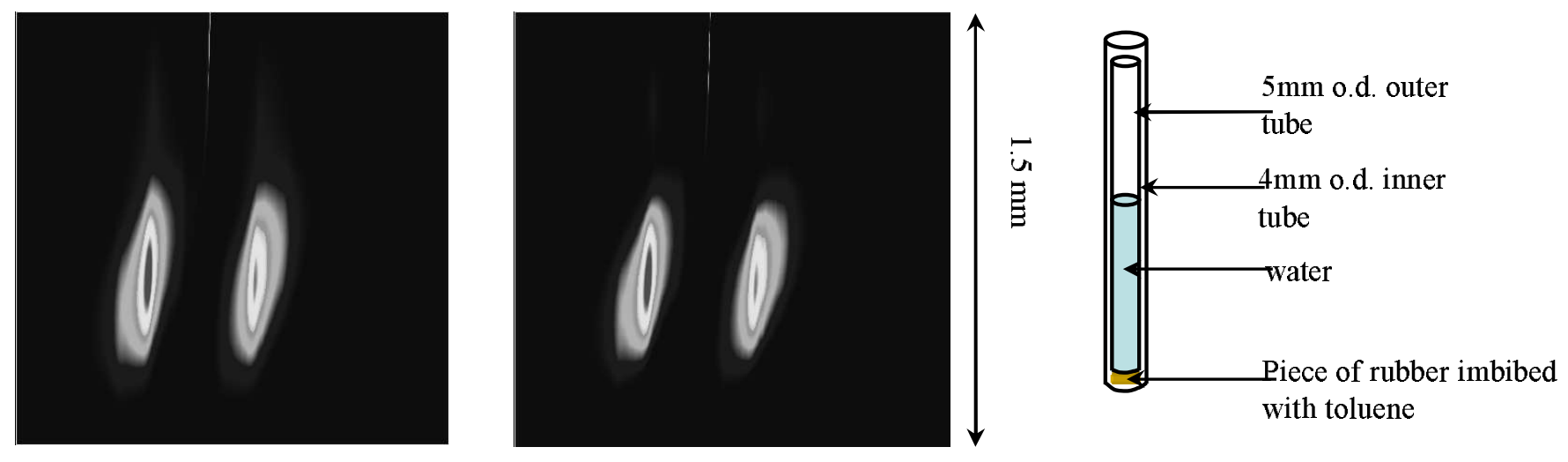

Figure 7 


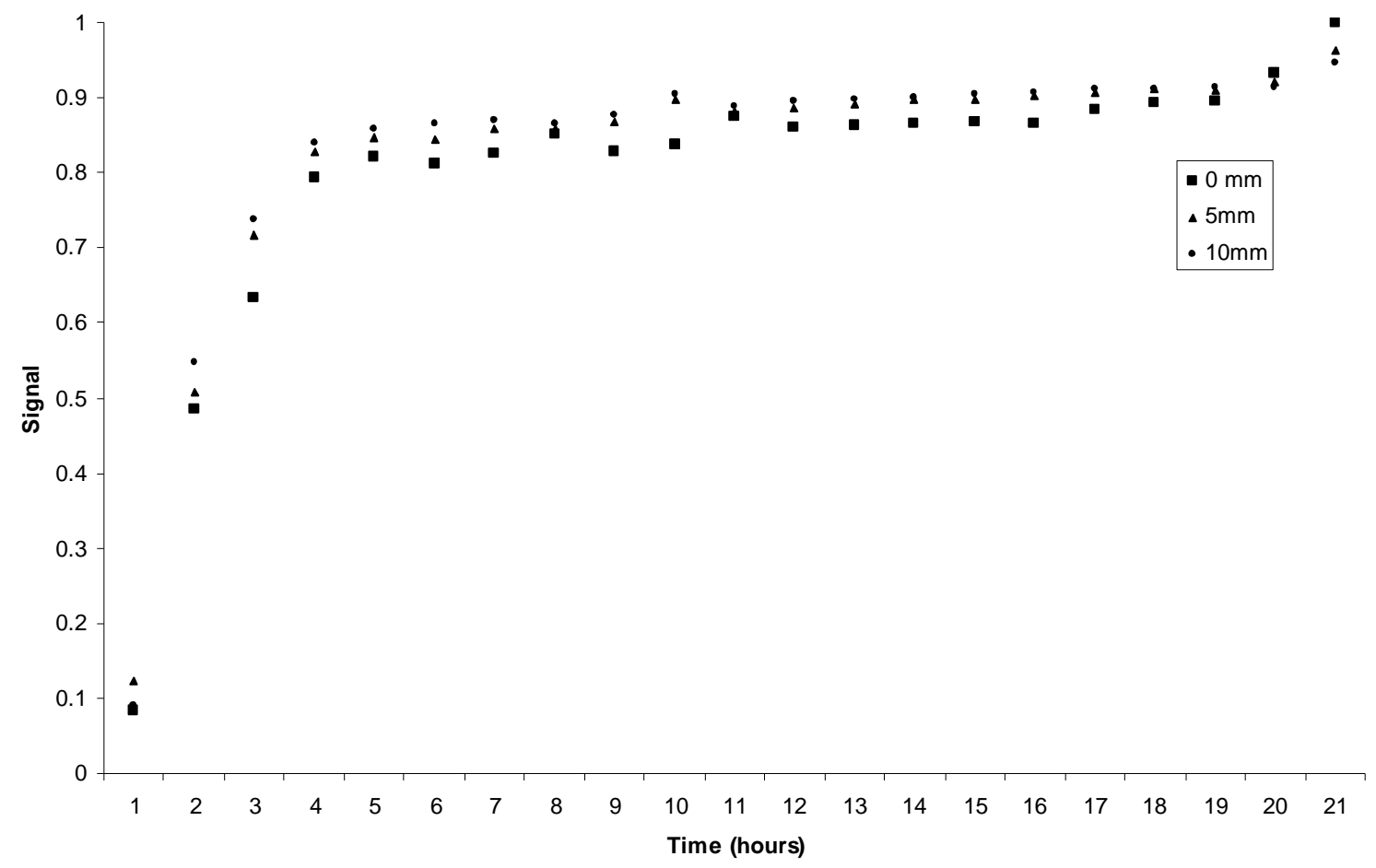

Figure 8 